\title{
Abdominal pain and swelling
}

\section{Scott Kilpatrick}

\section{An approach to the acute abdomen}

Presentation of the dog with acute abdominal pain is common in veterinary practice (Figure 25.1). The general causes of abdominal pain include: distension of a hollow viscus or organ capsule; ischaemia; traction; and inflammation secondary to a variety of conditions (Figure 25.2).

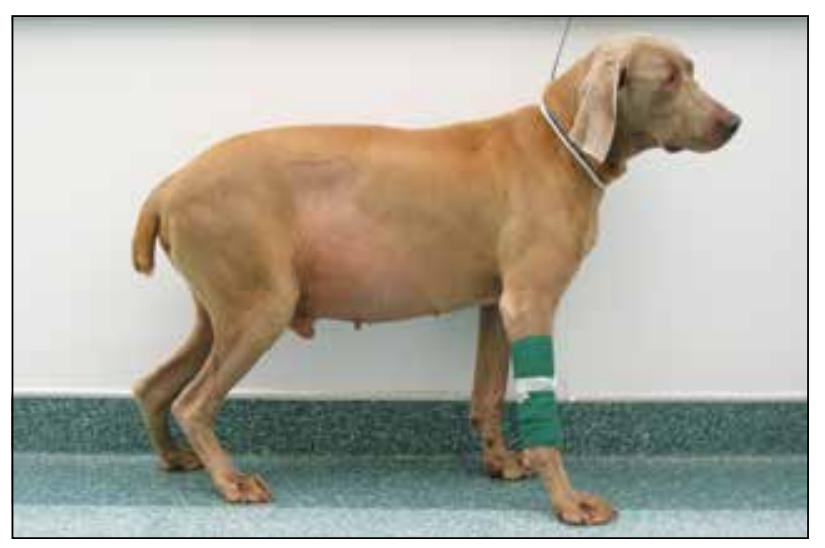

25.1

A 12-year-old male entire Weimaraner with abdominal distension due to an abdominal mass originating from a retained testicle.

\section{PRACTICAL TIPS}

- Not all life-threatening intra-abdominal problems are painful, especially in profoundly sick animals with mental depression

- Extra-abdominal sites of pain may present as an apparent acute abdomen. This happens most commonly with spinal pain

\section{WARNING}

All dogs with an acute abdomen potentially have a life-threatening condition that may require rapid surgical intervention. Although a thorough diagnostic work-up should be completed at some stage, emergency treatment must take priority

\section{Gastrointestinal system}

- Gastrointestinal perforation

- Gastric dilatation-volvulus

- Gastrointestinal dehiscence

- Intestinal obstruction

- Gastrointestinal neoplasia

Reproductive system

- Pyometra/uterine rupture

- Dystocia

- Ovarian cyst

- Ovarian neoplasia

- Prostatic abscess

- Prostatic neoplasia

- Testicular torsion

Hepatobiliary system

- Hepatic abscess

- Acute hepatitis

- Hepatic trauma/rupture

- Hepatobiliary neoplasia

- Cholangiohepatitis

- Biliary obstruction/rupture

Pancreatic system

- Acute pancreatitis

- Pancreatic abscess

- Pancreatic neoplasia

Urinary system

- Urinary calculi (renal, ureters, bladder, urethra)

- Pyelonephritis

- Renal neoplasia

\section{Haemopoietic system}

- Splenic mass

- Splenic rupture

- Splenic torsion

Peritoneum and abdominal wall

- Blunt trauma to abdominal wall

- Penetrating trauma to abdominal wall

- Septic peritonitis

- Chemical peritonitis (bile, urine, pancreatic)

- Mesenteric volvulus

Common causes of an acute abdomen 


\section{Initial evaluation and stabilization}

On initial presentation, a primary examination (see also Chapters 8 and 9) should include evaluation of:

- Level of consciousness

- Airway

- Breathing

- Circulation.

This should be completed within $30-60$ seconds. A very brief history should be obtained at this time, but resuscitation should not be delayed in the critical patient while a complete history is obtained (Figure 25.3).

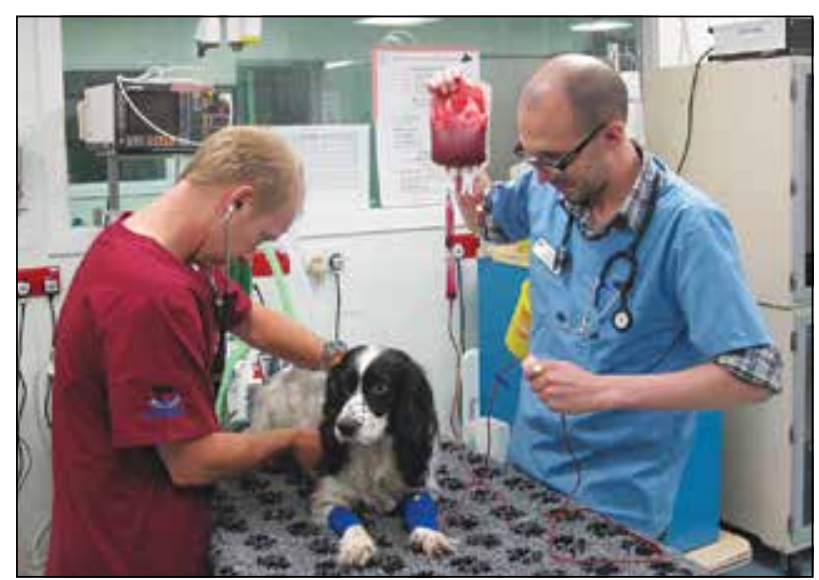

25.3

A 5-year-old male entire Cocker Spaniel receiving a blood transfusion after presenting with acute abdominal pain and abdominal distension due to a haemoabdomen (caused by a ruptured splenic haematoma). The dog initially presented collapsed, and fluid resuscitation was administered before providing transfusion support. The dog made a full recovery after surgical removal of the spleen.

The goal of any initial stabilization is to reverse the signs of shock and improve oxygen delivery to the cells.

- Oxygen and intravenous fluid therapy should be provided.

- Patients in extremis require rapid intubation and breathing support.

- Hypoglycaemic patients should be treated with intravenous glucose.

- Analgesia is an important part of initial stabilization. Opioids are considered the most appropriate form of analgesia; however, their dose may need to be reduced due to the fact that critical care patients are more sensitive to the sedative and negative cardiorespiratory effects of these drugs.

- The use of corticosteroids remains controversial and should be avoided until a more definitive diagnosis is made.

\section{WARNING}

Non-steroidal anti-inflammatory drugs (NSAIDs) should be avoided due to the potentially negative effects on some abdominal organs in a compromised patient

\section{History}

Following stabilization, a complete and detailed history must be obtained from the owner to ascertain the possible causes of the acute abdomen. Important questions to ask include the following:

- Is the dog neutered?

- Is there the potential for exposure to toxins or dietary indiscretion?

- Has there been any change in the dog's appetite or water intake in the days preceding presentation?

- Is ingestion of a foreign body a possibility?

- Does the dog have any ongoing medical problems or has it had any major medical problems in the past?

- Is the dog receiving any medication, including over-the-counter drugs (e.g. NSAIDs, aspirin)?

- Is there any possibility of trauma?

- When was the dog last vaccinated?

- Has there been any vomiting or diarrhoea? If present, characterize

- Has the dog been urinating normally? If not, characterize.

The history of a patient presenting with an acute abdomen is often vague, but reported signs will frequently include depression, anorexia and vomiting. In other instances the history may be extremely suggestive of the underlying cause. The progression of the clinical signs can also help determine the urgency of diagnosing the underlying cause. Chronic abdominal pain that has remained relatively static in its progression is not usually an emergency, although deterioration could precipitate a crisis. A dog that has a chronic problem and has deteriorated rapidly, or an animal with an acute problem, warrants a more aggressive approach to define the underlying cause.

In many cases the patient's signalment may lead to a higher index of suspicion of the aetiology. For example:

- Parvoviral enteritis may be suspected in dogs with an uncertain vaccination history

- Intussusception is more common in young animals

- Male dogs are much more likely than females to suffer from urethral obstruction

- German Shepherd Dogs and Golden Retrievers (median age of affected dogs 10 years) have a higher incidence than other breeds of haemoabdomen associated with splenic neoplasia

- Large- and giant-breed dogs have a much higher incidence of gastric dilatation and volvulus (GDV) than smaller breeds

- Entire males are at much higher risk of severe prostatitis than neutered males

- Entire female dogs may develop pyometra.

\section{Physical examination}

A full physical examination should be performed, but with initial attention to the cardiovascular, respiratory, central nervous and renal systems. 


\section{PRACTICAL TIP}

Examination of the abdomen should occur after that of the thorax, to avoid inadvertently missing important findings that may present in other body systems. Additionally, examination of the abdomen may elicit pain and discomfort that may prevent further evaluation of the patient

\section{Examination of the abdomen}

1. Visual inspection of the external abdomen.

- Abnormalities detected can include: distension (e.g. caused by effusion, gastric dilatation or organomegaly); subcutaneous swelling (e.g. resulting from cellulitis associated with urine leakage); and bruising (e.g. associated with trauma or a coagulopathy).

- Careful observation of the periumbilical area for evidence of reddening or haemorrhage may lead to a diagnosis of haemoabdomen.

- Any obvious wounds should also be carefully noted.

2. Auscultation of gastrointestinal sounds.

- Ingestion of toxins, acute intestinal obstruction and gastroenteritis may cause an increased frequency and character of gut sounds.

- Conditions such as ileus, anorexia, chronic intestinal obstruction and abdominal effusions can cause decreased frequency and character of gut sounds.

- The abdomen should be listened to over a period of 2-3 minutes to determine whether gastrointestinal sounds are actually absent.

- Abnormal sounds are not pathognomonic for any particular disease process.

- Note: Auscultation should precede palpation since palpation can cause gut sounds to diminish.

3. Percussion of the abdomen.

- Hands are placed lightly on either side of the abdominal wall with the animal standing. One hand is used to give a light but firm tap to one side of the abdomen, while the other feels for the presence of a ripple effect as the fluid in the abdomen is pushed towards the opposite abdominal wall.

- This may reveal a fluid wave in dogs with abdominal effusion, or tympanic abdominal sounds in dogs with gaseous gastric distension.

- Note: Occasionally a fluid wave can be elicited in animals without abdominal effusion. Obese animals, as well as those with a full bladder or fluid-filled viscus (e.g. pyometra) are good examples of this.

\section{Palpation.}

- The abdomen should be palpated in a systematic fashion.

- Superficial and gentle palpation should be carried out to help to localize pain.

- Animals may react vigorously and become tense, 'guarding' their abdomen in response to superficial palpation.

- Other more violent reactions may include vocalizing, groaning, yelping, vomiting, or attempting to bite the person performing the examination.

- Note: Some animals without abdominal pain will resent abdominal palpation and guard their abdomen. In these situations stroking the animal or spending some time building up the intensity of palpation will facilitate examination.

- The examination should, where appropriate, proceed to deeper palpation.

- Some conditions may be associated with localized pain (e.g. cranial abdominal pain with pancreatitis and caudal abdominal pain with prostatic disease).

- Palpation of the cranial abdomen in deepchested dogs is improved by elevating their thoracic limbs above floor level.

- Foreign bodies may be directly palpated and bunched up intestines may suggest a linear foreign body.

- A thick tubular structure may indicate a foreign body or intussusception.

- Septic peritonitis in dogs is usually, but not always, associated with severe, diffuse abdominal pain.

- Uroabdomen and bile peritonitis may or may not be painful.

- In many cases, sequential examination of the abdomen allows accurate assessment of the clinical condition.

\section{Other examinations required}

- Rectal temperature should always be taken, but note that in a dog with severe hypoperfusion the rectal temperature may not be elevated even if there is a raised core body temperature.

- The vulva of an intact bitch should be examined for any evidence of discharge, as seen in the case of an open pyometra.

- The oral cavity should always be examined for evidence of ingestion of caustic substances.

- Examination per rectum should always be performed to assess the caudal to middle pelvis, pelvic urethra and characteristics of the faeces. In male dogs the prostate gland should always be palpated.

\section{Diagnostic investigations Laboratory tests}

Minimum database: Blood tests including packed cell volume (PCV), total solids (TS) via refractometer, blood urea nitrogen (BUN; by dipstick) and glucose should all be part of a minimum database (Figure 25.4). A urine sample should also be obtained at the earliest opportunity; for the measurement of specific gravity, the urine sample should be obtained before any fluid therapy.

The minimum database is unlikely to provide a definitive diagnosis, but may be helpful in prioritizing differential diagnoses and further testing. For example:

- A low TS value in the face of a normal PCV should prompt a search for haemorrhage or severe vasculitis. In acute haemorrhage, the level of TS falls but splenic contraction blunts the expected 

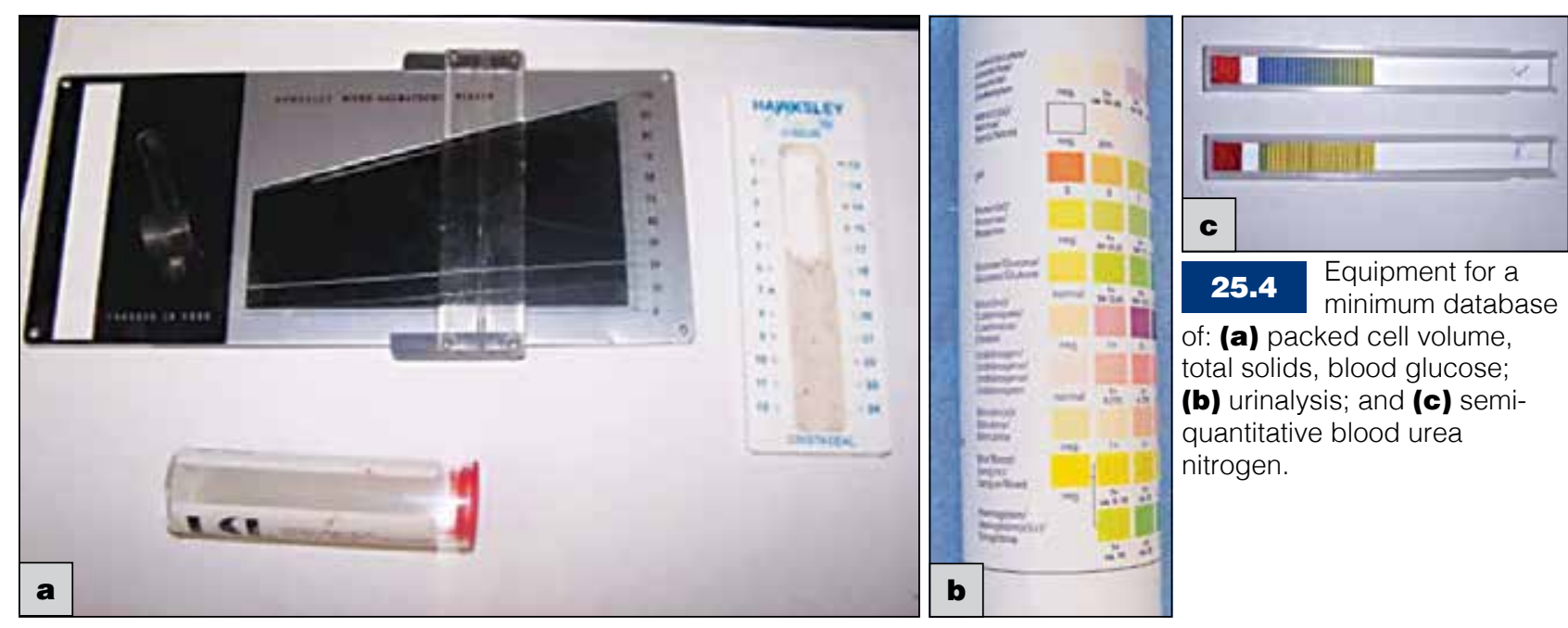

of: (a) packed cell volume, total solids, blood glucose;

(b) urinalysis; and (c) semiquantitative blood urea nitrogen.

fall in PCV. In patients with vasculitis, most commonly due to septic peritonitis, protein loss into the abdomen causes a fall in TS levels without affecting the PCV

- Decreased blood glucose is often associated with sepsis and warrants an aggressive approach to find the underlying cause of the acute abdominal pain, particularly if septic peritonitis might be present

- Dipstick BUN provides an estimate of azotaemia Increased BUN may be due to prerenal, renal or postrenal causes. Increased BUN may also be noted in animals with acute abdominal pain caused by pyelonephritis, or urethral or ureteral obstruction.

\section{Further tests:}

- Ideally, venous blood gas and electrolyte measurements should be carried out along with serum biochemistry as part of a more extensive investigation.

- A complete blood count should be performed, with microscopic evaluation of a blood smear in order to complete a manual differential count, estimate the number of platelets, and evaluate both white and red cell morphology.

- Coagulation parameters should be evaluated in patients with suspected liver disease, systemic inflammatory response syndrome (SIRS) or sepsis.

- Serum amylase and lipase are sometimes used as indicators (albeit neither sensitive nor specific) of pancreatitis in canine patients. In cases of acute pancreatitis the preferred test that is specifically useful and sensitive is the canine pancreatic lipase immunoreactivity (cPLI) assay; the sample may be sent to a laboratory for a quantitative result, but an in-house test for positive versus negative is useful for an immediate answer.

\section{Abdominal imaging}

Abdominal imaging should be performed in most cases of acute abdominal pain, as soon as the patient is sufficiently stable.

Radiography: Generally, two orthogonal views of the abdomen (lateral and dorsoventral) should be taken. A systematic and detailed review of all abdominal and adjacent structures should be performed.
Radiographs should be checked for:

- Radiopaque foreign bodies

- Intestinal dilatation

- Gastric size, position and content

- Liver and kidney size

- Uterine size

- Abnormal abdominal objects (e.g. cystoliths, Figure 25.5) or masses.

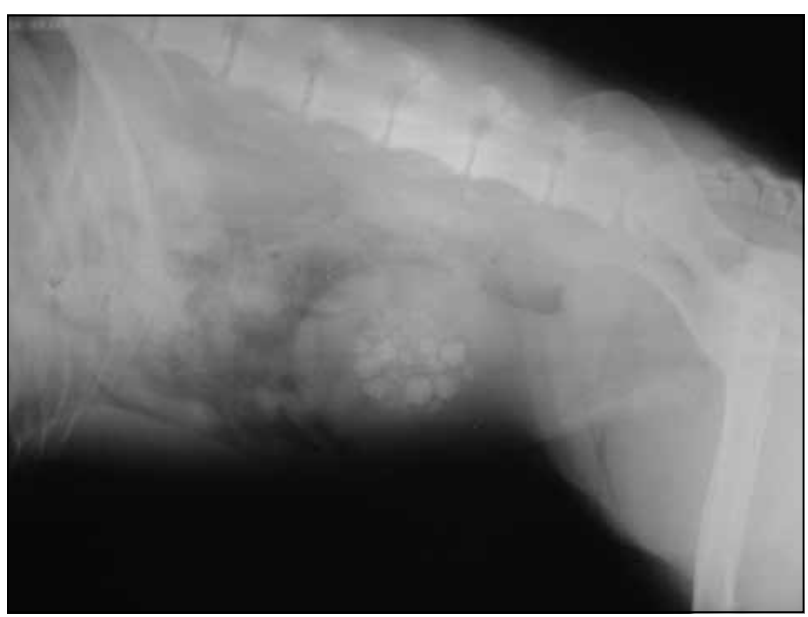

25.5 Lateral radiograph showing obvious bladder stones. This 3-year-old neutered Bichon Frisé bitch presented with dysuria and severe abdominal pain. Cystotomy was performed to remove the bladder stones that were later determined to be composed of calcium oxalate.

Loss of abdominal detail on plain abdominal radiographs may be due to lack of fat in the abdomen (in puppies or very thin animals), free abdominal fluid or a large abdominal mass. Free gas in the abdomen of a patient that has not undergone recent abdominal surgery or had a penetrating injury is consistent with bowel rupture or perforation.

Gastric distension with a normally positioned stomach is likely to be due to dilatation. If there is displacement with gastric dilatation, volvulus is likely. In cases of GDV, the stomach appears compartmentalized with band-like soft tissue opacities between gas-filled segments.

Segmental gaseous or fluid-filled distension of the small bowel suggests an intestinal obstruction. The 
normal diameter of the small intestine in the dog is approximately 2-3 times the width of a rib, or less than the width of an intercostal space. Additionally, all of the small intestinal loops should have a similar diameter, and it is abnormal for one segment to be $50 \%$ larger than other portions. Localized small intestinal distension is not always a definitive finding for intestinal obstruction but should prompt further investigation if an obvious foreign body is not evident (Figure 25.6). One option is to repeat radiography 3 hours later. If the intestine remains distended in the same position, this would indicate obstruction.

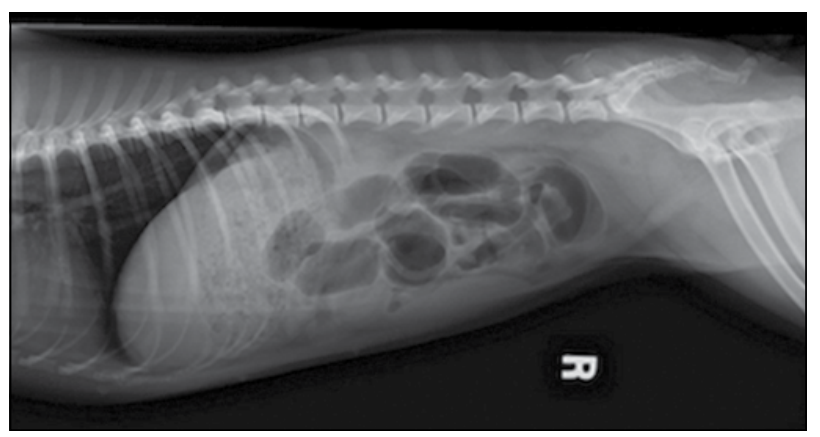

25.6 Lateral abdominal radiograph showing multiple loops of distended intestine in a 2-year-old male neutered Border Terrier. No obvious obstruction was seen; however, a sock foreign body was later removed surgically.

Contrast radiography may be necessary in some dogs with an acute abdomen, particularly those with partial gastrointestinal obstruction. Contrast radiography may be contraindicated in dogs with frequent vomiting, due to the high risk of aspiration pneumonia. The use of water-soluble contrast agents is often advocated if gastrointestinal perforation is suspected, to avoid barium contamination of the peritoneal cavity.

Ultrasonography: Ultrasound examination is more sensitive than radiography for examining abdominal masses and the presence of free fluid within the abdominal cavity. The pancreas, liver, kidneys and prostate gland can be evaluated more fully, and ultrasonography is one of the best ways of detecting pancreatitis and pancreatic masses. While pyometra may often be diagnosed radiographically, ultrasonography is a sensitive method for confirming a suspected and more subtle presentation of pyometra.

\section{An approach to the swollen abdomen}

Animals can develop a swollen abdomen for many reasons (Figure 25.7) and this can present as an emergency.

- Fluid: free fluid in abdomen; fluid inside organs; fluid in cysts

- Wear of abdominal wall muscles: hyperadrenocorticism

- Tissue: pregnancy; organ enlargement; neoplasia; fat; granuloma

- Faeces: megacolon; obstipation

- Gas: gastric dilatation-volvulus; obstruction or ileus of the gastrointestinal tract; post-surgical; rupture of the gastrointestinal tract; bacterial peritonitis

\section{Definitions}

- Peritoneal effusion is the abnormal accumulation of fluid in the peritoneal cavity and is not diagnostic in itself, but is a clinical sign of disease. It can result from the accumulation of transudative or exudative fluid, chylous effusions, blood, urine or bile

- Ascites is defined as an accumulation of serous fluid in the peritoneal cavity and is usually reserved for a transudate that is associated with liver disease or right-sided heart failure

\section{Diagnostic investigations}

As with any clinical problem, it is important to adopt a methodical approach (Figure 25.8).

\section{Ultrasonography}

One of the most straightforward ways of confirming the presence of free fluid in the abdominal cavity is with ultrasonography. Abdominal radiography is less useful in patients with large volumes of abdominal fluid, due to loss of intra-abdominal contrast. In some cases the presence of free fluid will be very obvious, and hypoechoic sharp angles will become immediately apparent once the ultrasound probe is placed on the patient (Figure 25.9).

Adopting a more structured 4-point FAST (focused assessment with sonography for trauma) scan (see QRG 25.1) will pick up on more subtle abdominal effusions (Figure 25.10). These scans can be performed very quickly and with no expert knowledge of ultrasonography needed. This is a procedure that can be carried out simultaneously with other interventions and can be repeated with little cost to the client.

\section{Laboratory tests}

Analysis of free abdominal fluid is a vital part of the evaluation of any animal with an acute/swollen abdomen. Abdominocentesis (see QRG 25.2), with or without ultrasound guidance, is a quick and easy way of retrieving abdominal fluid. It is unusual for complications to occur due to abdominocentesis and there are few contraindications to performing this procedure. Caution is advised, however, when a coagulopathy or thrombocytopenia is possible, or if there is organomegaly, or adhesions from previous surgery. A volume of at least $5-6 \mathrm{ml}$ of free abdominal fluid per kilogram needs to be present for successful abdominocentesis. Diagnostic peritoneal lavage (see QRG 25.3) may be necessary in cases where fluid is not obtained.

Once abdominal fluid has been obtained, it is important to classify this to allow a more accurate diagnosis to be made. Analysis of the abdominal fluid should include: gross examination; measurement of PCV; TS by refractometer; and total nucleated cell count. Cytological examination is also very important. Biochemical and microbiological analysis are appropriate in certain cases. Classification of the main types of abdominal effusion is summarized in Figure 25.11; some further details are given below. 


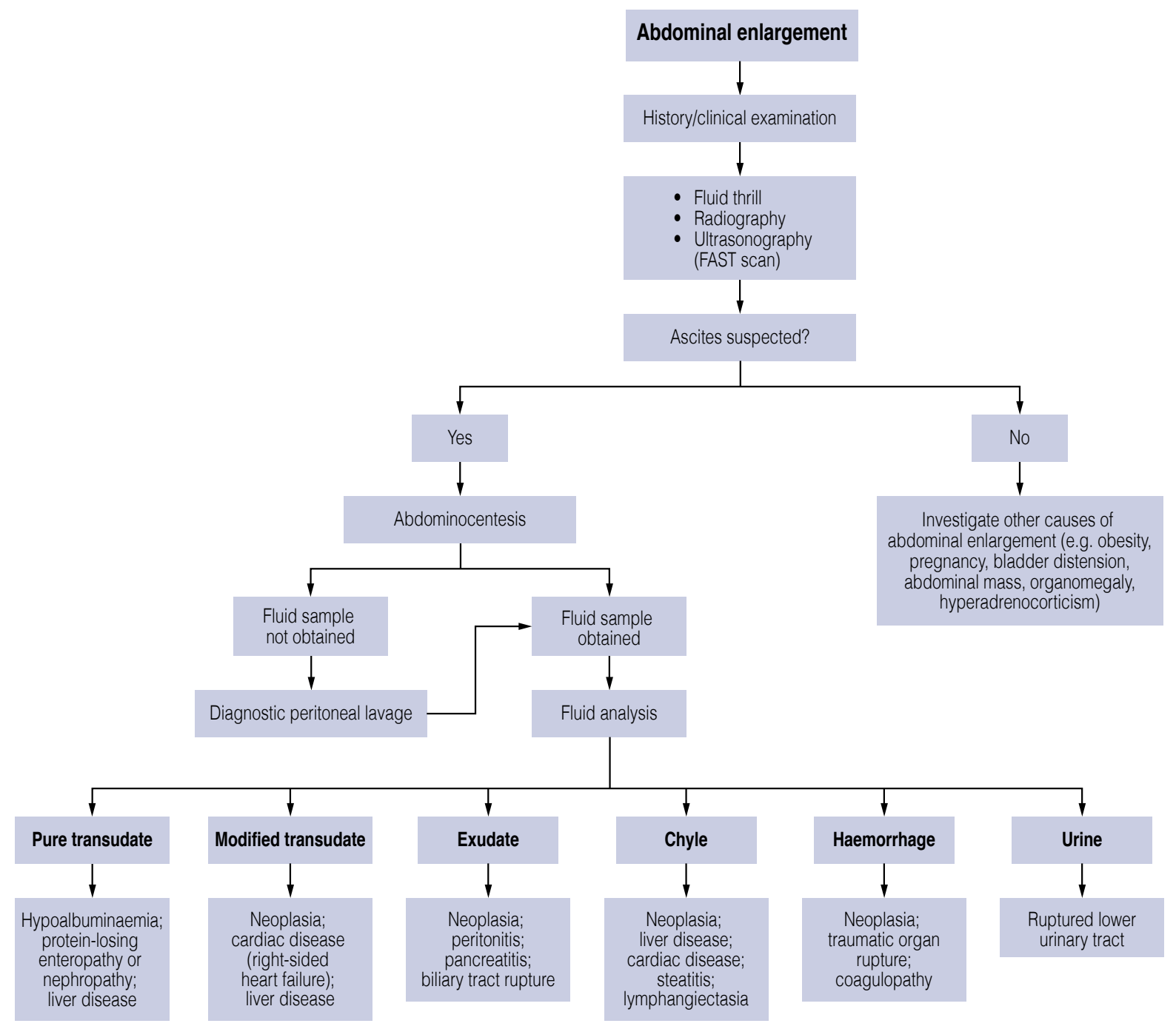

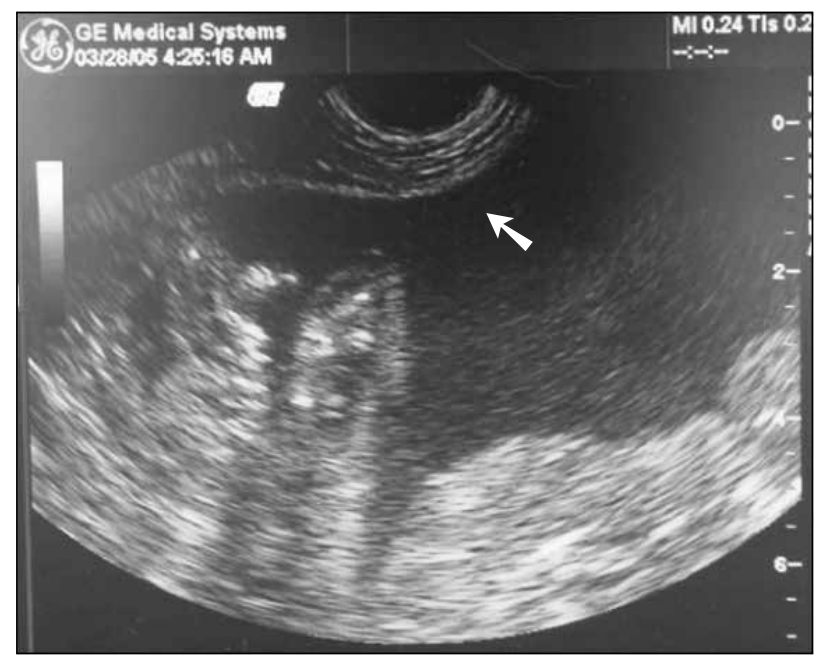

25.9
Obvious free fluid (arrowed) within the abdominal cavity detected using ultrasonography.

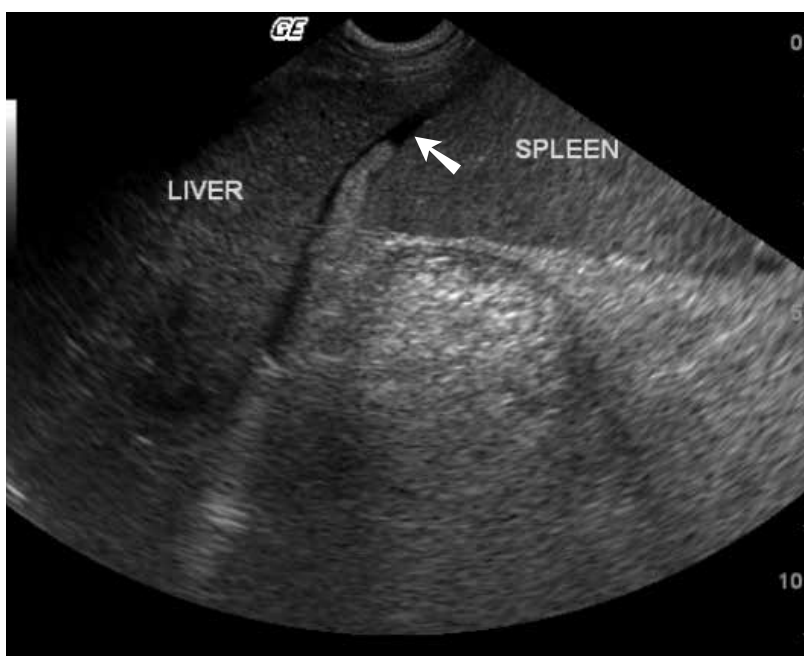

25.10 Subtle area of free fluid (arrowed) between the liver and spleen. 


\begin{tabular}{|c|c|c|c|c|c|}
\hline Characteristic & Transudate & Modified transudate & Exudate & Chylous effusion & Haemorrhagic effusion \\
\hline Appearance of fluid & Clear, colourless & $\begin{array}{l}\text { Clear, straw-coloured or } \\
\text { blood-tinged }\end{array}$ & Turbid & $\begin{array}{l}\text { Milky or pinkish } \\
\text { opaque fluid }\end{array}$ & $\begin{array}{l}\text { Appears similar to } \\
\text { peripheral blood }\end{array}$ \\
\hline Total protein ( $\mathrm{g} / \mathrm{l})$ & $<25$ & $>25$ & $>25$ & $25-60$ & $>30$ \\
\hline Specific gravity & $<1.015$ & $1.015-1.025$ & $>1.025$ & $>1.025$ & $>1.025$ \\
\hline $\begin{array}{l}\text { Nucleated cells } \\
\left(\times 10^{9} / \text { litre }\right)\end{array}$ & $<1$ & $1-7$ & $>5$ & $0.25-20$ & $>1$ \\
\hline $\begin{array}{l}\text { Predominant cell } \\
\text { type }\end{array}$ & $\begin{array}{l}\text { Primarily } \\
\text { mesothelial cells } \\
\text { and macrophages }\end{array}$ & $\begin{array}{l}\text { Increasing numbers of } \\
\text { neutrophils and small } \\
\text { lymphocytes, macrophages } \\
\text { and possibly neoplastic cells }\end{array}$ & $\begin{array}{l}\text { Primarily neutrophils } \\
\text { (non-degenerate, or } \\
\text { degenerate if septic) }\end{array}$ & $\begin{array}{l}\text { Small lymphocytes, } \\
\text { neutrophils, } \\
\text { macrophages }\end{array}$ & $\begin{array}{l}\text { Large proportion of red } \\
\text { blood cells and usually } \\
\text { without platelets }\end{array}$ \\
\hline Other features & $\begin{array}{l}\text { With time pure } \\
\text { transudates will } \\
\text { become modified } \\
\text { due to irritation of } \\
\text { the mesothelium }\end{array}$ & $\begin{array}{l}\text { Most modified transudates } \\
\text { are caused by neoplasia or } \\
\text { heart failure }\end{array}$ & $\begin{array}{l}\text { Can be classified as } \\
\text { septic or non-septic }\end{array}$ & $\begin{array}{l}\text { Contains high levels } \\
\text { of triglycerides }\end{array}$ & $\begin{array}{l}\text { This type of effusion does } \\
\text { not normally clot }\end{array}$ \\
\hline
\end{tabular}

\section{- Transudates:}

- Usually characterized by having low cellularity, low specific gravity (SG) and low total protein

- Occur secondary to decreases in oncotic pressure due to hypoalbuminaemia (proteinlosing enteropathy, protein-losing nephropathy, liver failure) or an increased hydrostatic pressure (portal hypertension, right-sided heart failure)

- Not normally associated with acute abdominal pain.

\section{Modified transudates:}

- Contain more protein and cells than pure transudates

- Usually occur due to an increased hydrostatic pressure, or increased vascular permeability in the early stages of inflammatory disease

- Often caused by neoplastic disease.

\section{- Exudates:}

- Highly cellular and can be septic or non-septic:

- The presence of toxic degenerate neutrophils with intracellular bacteria is indicative of septic peritonitis (Figure 25.12)

- Non-septic exudates are characterized by the presence of non-degenerate neutrophils and the absence of bacteria

- Unfortunately these cytological changes are not always present and further testing may be needed:

- A glucose level can be taken from the effusion. An abdominal fluid glucose level of $<2.8 \mathrm{mmol} / \mathrm{l}$ has been shown to indicate septic peritonitis unequivocally, since bacteria metabolize glucose present in the effusion

- Another method is to compare the blood/effusion levels of lactate. If the lactate concentration in the peritoneal effusion is $>2.5 \mathrm{mmol} / \mathrm{l}$ and higher than the blood lactate level, a septic effusion is likely.

\section{- Chylous effusion:}

- Many effusions can be run through blood analysers in the same way as a blood sample

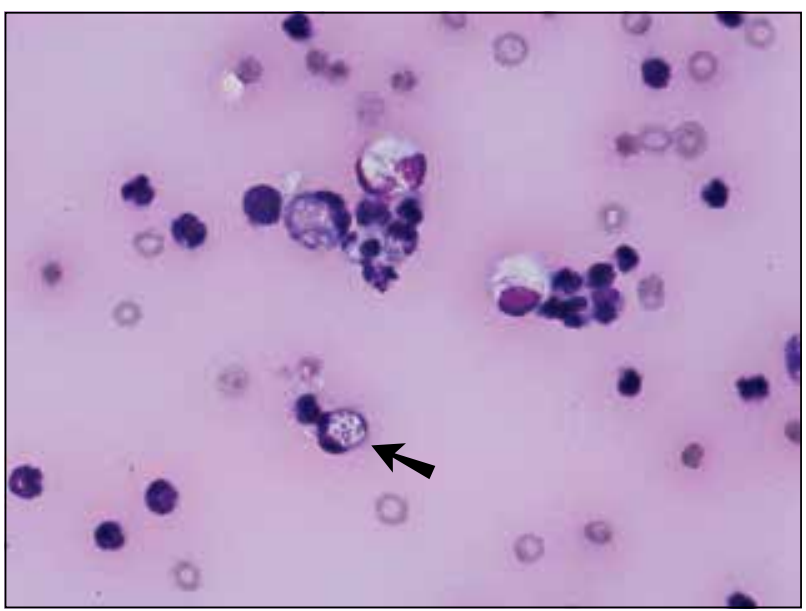

25.12 The presence of intracellular bacteria (arrow) is an indication of septic peritonitis. (H\&E stain; original magnification X100)

- The triglyceride level in a chylous effusion is usually 2-3 times higher than in serum, while cholesterol is lower in the effusion than in serum

- The most common causes of chylous effusions are rupture of a lymph vessel due to neoplasia, right-sided heart failure and lymphoproliferative disease.

\section{- Bilious effusions:}

- Biliary rupture will rapidly create a modified transudate that progresses to an exudate later in the disease process

- Bile effusions occur secondary to rupture of the bile duct or gall bladder. This happens secondary to trauma, cholelithiasis, pancreatitis, or necrotizing cholecystitis

- If bile peritonitis is suspected, total bilirubin should be measured. If the bilirubin concentration in the abdominal fluid is greater than that in the serum, bile peritonitis is confirmed. Normally, there should not be any bilirubin present in abdominal fluid. The fluid is often green and may contain bilirubin crystals.

\section{- Uroabdomen:}

- Fluid from a patient with uroabdomen can range from a modified transudate to an exudate 
- Uroabdomen can result from trauma or from iatrogenic causes (e.g. careless catheterization or cystocentesis). It is important to note that animals with ruptured bladders may still have palpable bladders and the ability to urinate

- If uroabdomen is suspected, the most useful abdominal fluid chemistry evaluation is creatinine. Urea nitrogen can also be measured but, because it is a smaller molecule, this diffuses rapidly and equilibrates with the plasma. A creatinine level in abdominal fluid that is twice that in serum is highly suggestive of uroabdomen

- Potassium levels are also higher in the abdominal fluid compared with blood

- Note: If the dog has been receiving intravenous fluids, there is the potential for misdiagnosis of uroabdomen in an azotaemic patient with ascites. This is because creatinine, BUN and potassium concentrations in the plasma will be actively diluted, whereas those in the abdomen will not.

\section{Haemoabdomen:}

- Haemorrhage into the abdomen may occur for many reasons, including trauma, neoplasia, coagulopathies and iatrogenic causes

- A PCV of $5 \%$ or higher in diagnostic peritoneal lavage fluid is suggestive of significant haemorrhage

- Cytologically, it may be difficult to differentiate acute haemorrhage from iatrogenically induced haemorrhage such as from inadvertent trauma of the liver or spleen. Platelets quickly aggregate, degranulate and disappear within an effusion, so their presence may be suggestive of iatrogenically induced haemorrhage.

- Malignant effusions:

- Malignant effusions are often a subtype of modified transudate or exudate that contain neoplastic cells

- Care should be taken in interpreting malignancy on cytology due to normal mesothelial cells displaying criteria that could be mistaken for neoplasia. Review by a pathologist is essential.

\section{Surgical management}

One of the most challenging decisions regarding dogs with acute abdominal pain or swelling is deciding whether prompt surgery is indicated.

\section{Indications for prompt surgery}

- Abdominal abscess

- Abdominal wall perforation

- Bile peritonitis

- Free abdominal gas (not associated with previous surgery or invasive procedures)

- Gastric dilatation-volvulus

- Intestinal foreign body causing pain or bowel obstruction

- Intestinal obstruction

- Ischaemic bowel

- Mesenteric volvulus

- Persistent abdominal haemorrhage

- Septic peritonitis

- Uroperitoneum

In some situations the decision to undertake surgery may not be straightforward.

\section{WARNING}

Even if prompt surgery is indicated, the immediate necessity is to stabilize the patient

\section{References and further reading}

Holt D and Brown D (2007) Acute abdominal and gastrointestinal surgical emergencies. In: BSAVA Manual of Canine and Feline Emergency and Critical Care, ed. LG King and A Boag, pp.174-191. BSAVA Publications, Gloucester

Kinns J (2011) Abdomen. In: BSAVA Manual of Canine and Feline Ultrasonography, ed. F Barr and L Gaschen, pp.72-84. BSAVA Publications, Gloucester

Lisciandro GR, Lagutchik MS, Mann KA et al. (2009) Evaluation of an abdominal fluid scoring system determined using abdominal focused assessment with sonography for trauma in 101 dogs with motor vehicle trauma. Journal of Veterinary Emergency and Critical Care 19, 426-437

O'Brien R and Barr F (2009) BSAVA Manual of Canine and Feline Abdominal Imaging. BSAVA Publications, Gloucester 


\section{QRG 25.1 FAST scan}

\section{Positioning and preparation}

- Right lateral recumbency is recommended, as this is also the best position for abdominocentesis, electrocardiography and echocardiography. However, this procedure can be carried out in a standing animal.

- One of the benefits of this procedure is that it can often be carried out in the conscious patient.

\section{Technique}

Placing the ultrasound probe on the four areas shown and scanning widely in both sagittal and transverse planes gives the best chance of detecting more subtle fluid accumulation (see Figure 25.10).

\section{Limitations}

- There is always the possibility of false-positive or false-negative results. For example, the gall bladder and common bile duct can

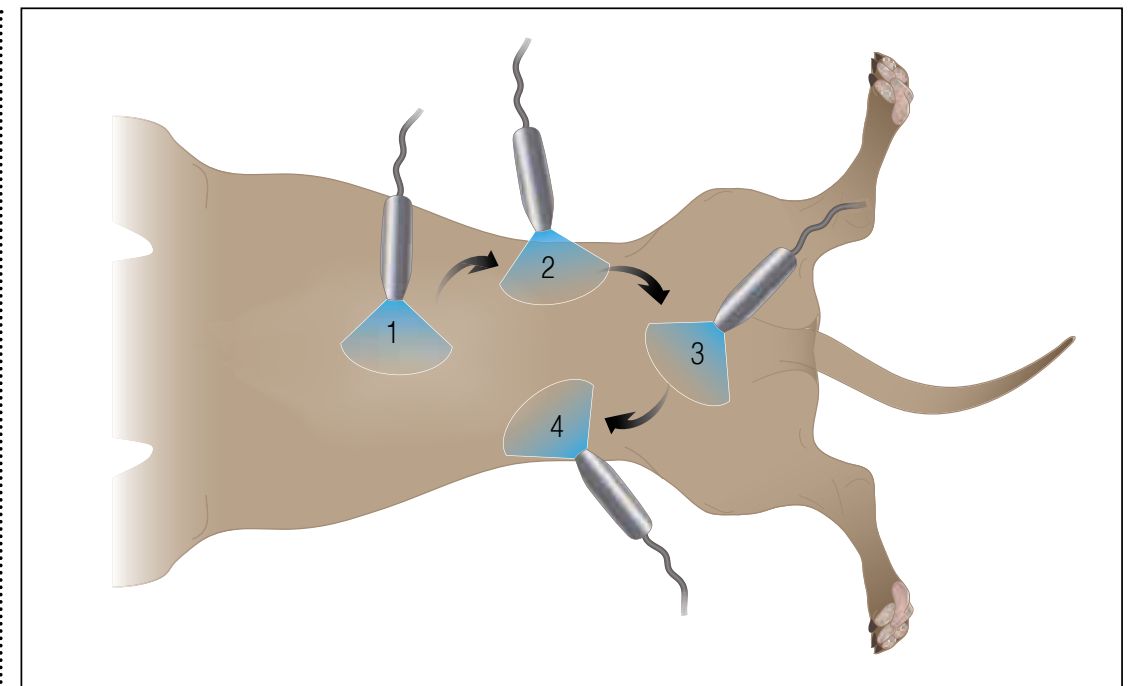

Four-point ultrasound assessment for free abdominal fluid. 1 = diaphragmaticohepatic; 2 = splenorenal; 3 = cystocolic; 4 = hepatorenal. (Redrawn after Lisciandro et al., 2009)

appear as hypoechoic sharp angles, similar to free fluid, depending on

the plane of imaging.

- If in doubt, the scan should be repeated on a regular basis to detect any changes.

\section{QRG 25.2 Abdominocentesis}

\section{Positioning and preparation}

- Sedation may or may not be required.

- The dog should be restrained in right lateral recumbency and it might be worth emptying the patient's bladder before the procedure.

- The abdomen should be clipped and prepared as for a non-surgical procedure.

\section{Equipment}

A $5 \mathrm{ml}$ syringe, collection tubes (EDTA, plain and sterile) and some microscope slides should be prepared.

Use an 18-22 G, 2.5-3.75 cm needle or over-the-needle catheter.

\section{Technique}

Abdominocentesis can be performed by a single centesis or using a fourquadrant approach.
The site for single abdominocentesis is a point $1 \mathrm{~cm}$ lateral and to the right of the ventral midline and $1-2 \mathrm{~cm}$ caudal to the umbilicus.

2 Once the needle has been inserted 2 through the skin and abdominal wall, allow fluid to drip from the needle (or catheter with needle removed) into a tube, or gently aspirate with a $2-5 \mathrm{ml}$ syringe. Unless there is a large volume of free fluid it is preferable to allow it to drip from the needle hub, rather than aspirating, to avoid sucking omentum or viscera into the needle.

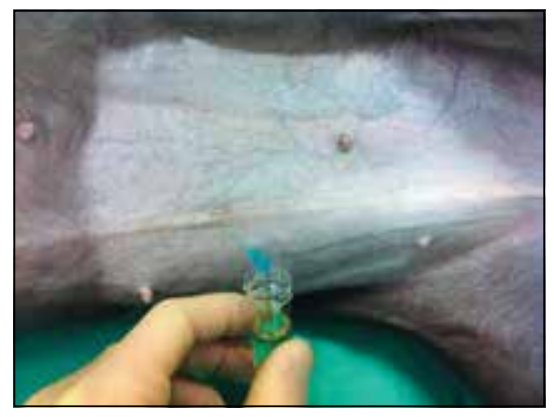

If fluid is not obtained from the first site, repeat the procedure in the three remaining sites.

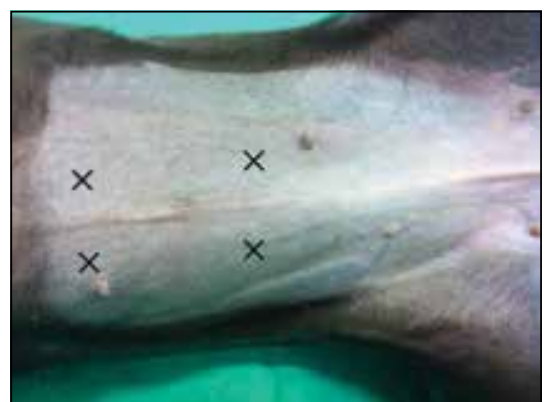

Collect fluid into an EDTA tube for cytology and cell count, and into a plain tube for culture and biochemical analysis.

5

\section{Make several air-dried smears.}




\section{QRG 25.3 Diagnostic peritoneal lavage}

\section{Positioning and preparation}

Positioning and preparation is as for abdominocentesis (see QRG 25.2).

However, a higher proportion of patients will need to be sedated for diagnostic peritoneal lavage. Local anaesthetic is infiltrated into the ventral midline, just caudal to the umbilicus.

\section{PRACTICAL TIP}

As diagnostic peritoneal lavage introduces fluid in to the peritoneal cavity, diagnostic imaging should be carried out before this procedure

\section{Technique}

Using a scalpel, make a small stab incision through the skin.

Introduce a large-bore 10-14 G over-the-needle catheter (pre- fenestrated) through the skin and body wall, aiming caudally towards the pelvis.

Remove the stylet, leaving the cannula in place.

Attach an extension set and a three-way tap.

5 Infuse $20 \mathrm{ml} / \mathrm{kg}$ of warmed isotonic - crystalloid fluid using gravity flow or gentle pressue.

Gently roll the patient (with the catheter in place if possible) and wait for 20-30 minutes.

7 Drain the fluid from the catheter:

- Use EDTA tubes for samples used to test total cell count and cytology

- Use plain tubes for samples for total protein and any other biochemical tests

- Fresh air-dried smears should be made
- Some fluid should be kept in a plain tube to be submitted for culture if necessary.

\section{Notes:}

- Only a very small amount of the infused volume will be retrieved (usually only 1-2 ml). Any remaining fluid will be absorbed across the peritoneum.

- If the fluid obtained suggests that the gastrointestinal tract has been punctured, any hole should seal when the needle is removed.

\section{WARNING}

Stop the procedure if blood is aspirated. Place the blood in a glass tube and monitor for clot formation. Free blood from the abdominal cavity will not clot (due to lack of platelets) but blood from a vessel or organ will clot 